\title{
Helical buckling of thin rods: FE modelling
}

\author{
Marcin Kapitaniak ${ }^{1, *}$, Vahid Vaziri $^{1}$, and Marian Wiercigroch $^{1}$ \\ ${ }^{1}$ Centre for Applied Dynamics Research, School of Engineering, University of Aberdeen, Aberdeen, \\ AB24 3UE, Scotland, UK
}

\begin{abstract}
In this paper, we utilize the Finite Element (FE) method to model twisting of long thin rods and capture the bifurcation scenarios leading to helical buckling and various further post-buckling states. Since standard nonlinear beam elements do not account for nonlinearity in torsional mode, we derive a modified beam element, which allows to model complex torsional buckling bifurcation scenarios of a thin rod subjected to twisting load. A series of verification tests (static analysis with load stepping) of the developed code are performed to determine critical torsional buckling loads for various helical buckling modes and compared with ABAQUS FE simulation.
\end{abstract}

\section{Introduction}

The study of helical buckling of long thin rods represents an interesting field of research, which allows to gain a deeper insight into various post-buckling configurations and study interplay between them. Helical buckling represents a significant modelling challenge, which has been tackled in the past using analytical approaches $[1,5,6]$ that allowed to identify various post-buckling helical modes in long twisted and stretched/compressed rods. As the twisting moment acting on a rod increases, the trivial undeflected solution becomes unstable and bifurcates, leading to writhing of the rod, with a number of helical loops increasing with the torque, as shown in Figure 1(a). In this work, we utilize the Finite Element (FE) method to describe twisting of long thin rods and capture the bifurcation scenarios leading to helical buckling and various further post-buckling states.

\section{Nonlinear finite element beam}

In order to gain a deeper understanding into helical buckling of long rods, it is vital to have an efficient and robust finite elements, that can precisely capture the coupling between twisting and bending degrees of freedom. This can be achieved by modifying a 3D large deflection Euler-Bernoulli or Timoshenko beam elements [2], by including appropriate nonlinear strain components into stiffness matrix derivation. Let us consider a 3D Euler-Bernoulli beam element, with two nodes and six degrees-of-freedom per node $\left(x, y, z, \Phi_{x}, \Phi_{y}, \Phi_{z}\right)$, which can

\footnotetext{
*e-mail: marcin.kapitaniak@abdn.ac.uk
} 
be described in the displacement field as:

$$
\begin{array}{r}
Q_{1}=u(x)-z \frac{\partial w(x)}{\partial x}-y \frac{\partial v(x)}{\partial x}, \\
Q_{2}=-z \theta_{x}+v(x), \\
Q_{3}=y \theta_{x}+w(x),
\end{array}
$$

where $u(x), v(x), w(x)$ are beam displacements along $x, y, z$ axes and $\theta_{x}$ is the beam rotation around $x$ axis. In order to derive a stiffness matrix for the beam element, one needs to calculate the strain energy, which for isotropic materials is

$$
U_{i}=\frac{1}{2} \int\left(E\left(\epsilon_{x x}^{2}+\epsilon_{y y}^{2}+\epsilon_{z z}^{2}\right)+G\left(\epsilon_{x y}^{2}+\epsilon_{y z}^{2}+\epsilon_{z x}^{2}\right)\right) d V .
$$

Assuming, that the cross section of the beam does not change during deformation $\left(\epsilon_{y y}=\right.$ $0, \epsilon_{z z}=0, \epsilon_{y z}=0$, ), the strain energy during deformation becomes

$$
U_{i}=\frac{1}{2} \int E \epsilon_{x x}^{2}+G\left(\epsilon_{x y}^{2}+\epsilon_{z x}^{2}\right) d V
$$

The first term provides the standard linear and nonlinear strain energies and ensures coupling between bending and axial degrees of freedom of the beam. This part of the beam element derivations is well known and can be easily found in FE textbooks [2-4]. However, if one wants to include coupling between the torsional $\left(\Phi_{x}\right)$ and all other DOF, it is necessary to calculate strain energy associated with shear. This can be easily done using the large deflection definitions of strain, keeping only the necessary terms, as shown below:

$$
\begin{gathered}
\epsilon_{x x}=\frac{\partial Q_{1}}{\partial x}+\frac{1}{2}\left(\left(\frac{\partial Q_{1}}{\partial x}\right)^{2}+\left(\frac{\partial Q_{2}}{\partial x}\right)^{2}+\left(\frac{\partial Q_{3}}{\partial x}\right)^{2}\right), \\
\epsilon_{x y}=\frac{\partial Q_{1}}{\partial y}+\frac{\partial Q_{2}}{\partial x}+\left(\frac{\partial Q_{1}}{\partial x} \frac{\partial Q_{1}}{\partial y}+\frac{\partial Q_{2}}{\partial x} \frac{\partial Q_{2}}{\partial y}\right), \\
\epsilon_{z x}=\frac{\partial Q_{1}}{\partial z}+\frac{\partial Q_{3}}{\partial x}+\left(\frac{\partial Q_{1}}{\partial x} \frac{\partial Q_{1}}{\partial z}+\frac{\partial Q_{3}}{\partial x} \frac{\partial Q_{3}}{\partial z}\right) .
\end{gathered}
$$

Using the standard polynomial shape functions [3], we are able to obtain a linear and nonlinear stiffness matrices, by differentiating the strain energy $U_{i}$ with respect to nodal displacements of the beam element $\left(\mathrm{u}=\left[x_{1}, y_{1}, z_{1}, \Phi_{x 1}, \Phi_{y 1}, \Phi_{z 1}, x_{2}, y_{2}, z_{2}, \Phi_{x 2}, \Phi_{y 2}, \Phi_{z 2}\right]^{\mathrm{T}}\right)$ as:

$$
\mathbf{F}=\left(\mathbf{k}_{\text {lin }}+\mathbf{k}_{\text {nonlin }}\right) \mathbf{u}
$$

where $\mathbf{F}, \mathbf{k}_{\text {lin }}$ and $\mathbf{k}_{\text {nonlin }}$ are vector of nodal forces, linear and nonlinear stiffness matrices.

\section{Numerical modelling}

In order to perform a validation of the derived beam element, we need to make sure that the torsional buckling occurs for the right loading conditions. For that purpose, let us consider a steel beam of length, $L=20 \mathrm{~m}$ and circular cross-section of radis $r=0.025 \mathrm{~m}$, supported as shown in Fig. 1(b) and subjected to end torque $M_{\text {end }}$. Using the linear and nonlinear matrices derived in Section 2, the beam is discretized into 90 elements, meaning we have 91 nodes, each having six DOF per node. From the modal analysis conducted in ABAQUS for the same beam, we obtain first 3 critical buckling loads of $M_{\text {end }}: 15822 \mathrm{Nm}, 33716 \mathrm{Nm}$ and $52860 \mathrm{Nm}$. 
(a)

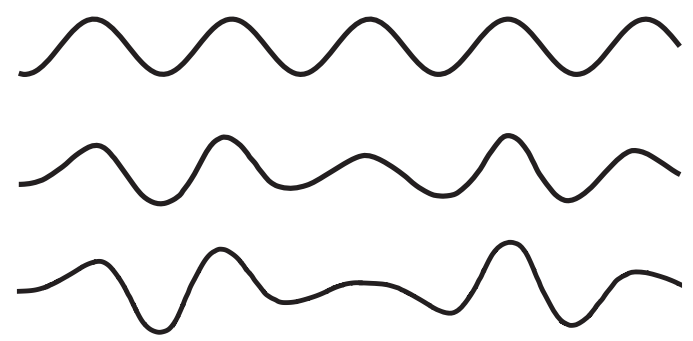

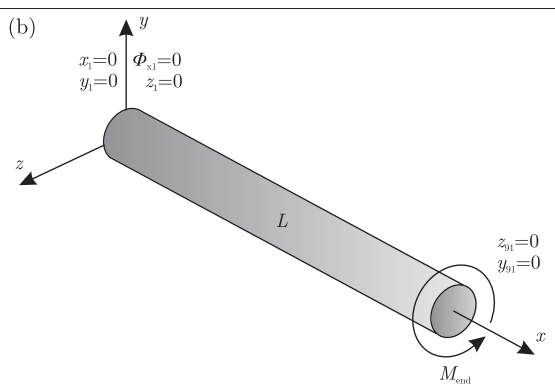

Figure 1. (a) Buckling states of a thin rod and (b) Configuration of the analysed beam showing the boundary conditions and loading applied.

In order to compute the critical buckling loads using the developed model, it is necessary to solve the static equation, $\mathbf{F}=\left(\mathbf{k}_{\text {lin }}+\mathbf{k}_{\text {nonlin }}\right) \mathbf{u}$, by finding the displacements corresponding to the applied loading, where only $M_{\text {end }}$ varies. This resulted in the system of 540 nonlinear equations, which was solved numerically by using an iterative Newton-Raphson method [4]. In order to pinpoint the buckling load, we started our analysis in the proximity of the expected value, changing the end torque value in small steps and using the previous step as an initial guess for the subsequent step. By monitoring the displacements of each node, we were able to determine changes indicating the onset to torsional buckling. In Figure 2 (a)-(f) we depict the set of displacements of the middle node (at $x=10 \mathrm{~m}$ ). As can be seen, in the beginning of the graphs, none of the displacements changes, until the end torque reaches value of 15814.28 $\mathrm{Nm}$ (marked as vertical dash lines in panels (a)-(f))). After this critical value, we observe a sudden change in all the coordinates $x_{45}, y_{45}, z_{45}, \Phi_{y 45}$ and $\Phi_{z 45}$. This indicates that torsional buckling occurred. From that point onwards, as the end torque increases we observe further increase in nodal displacements, that correspond to post buckling configuration. Note, that the qualitative shape of the beam does not change, just the amplitude in each node. In panels (b)-(c) and (e)-(f), we observe four different symmetric branches coexisting with each other, but qualitatively the post buckling shapes of the beam are the same, just the direction in which helix goes is different. Following the same principle we compute second and third buckling modes, observing that second torsional buckling occurs at $33712.5 \mathrm{Nm}$, while the third one at $52858.2 \mathrm{Nm}$ (both marked by dashed lines in Figure 2). This indicates an excellent agreement with ABAQUS simulation (quad FE elements), with absolute errors of $0.04 \%, 0.01 \%$ and $0.003 \%$ for first, second and third torsional buckling loads. The projections of the shapes of the beam at these three buckling points are depicted in Figure 3 (a)-(c), while corresponding shapes from ABAQUS are depicted next to the graphs. Note, that the scales are different, so only qualitative comparison of shape is possible. For simplicity, just two of the branches (marked in blue and orange), depicted in Figure 2 (a)-(f), are shown in this case. The other two branches are symmetric with respect to the origin.

Having established, that the proposed FE beam element captures the torsional buckling accurately, we are able to look into more details at the post-buckling parts of the curves depicted in Figure 2 (a)-(f). In panel (g), we depict the zoomed view of panel (b), where all three analyzed post-buckling scenarios A, B, C are marked in dark blue, red and green respectively. Looking at the first scenario, it is visible that as the load increases, so does the lateral deformation. This happens up to some point $(41734.2 \mathrm{Nm})$, when the first post buckling scenario disappears. This indicates that there is a region where both first and second post buckling scenarios coexist. Similar coexistence can be observed between scenarios $\mathrm{C}$ and $\mathrm{B}$, though the 

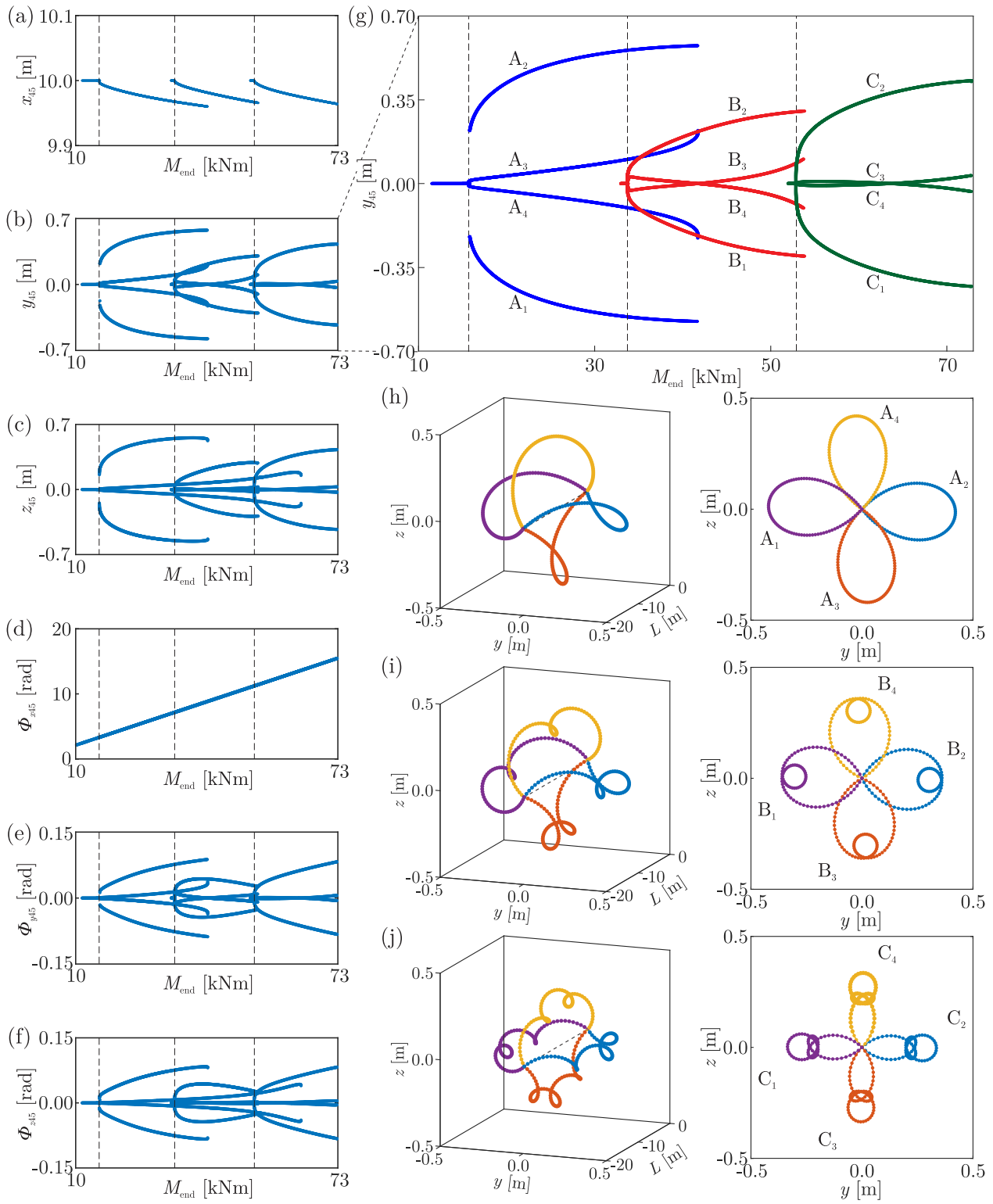

Figure 2. Post-buckling deformation of the beam, where panels (a)-(f) depict displacements $\left(x_{45}, y_{45}\right.$, $\left.z_{45}, \Phi_{x 45}, \Phi_{y 45}, \Phi_{z 45}\right)$ of the middle node of the beam as a function of end torque $M_{\text {end }} \cdot(\mathrm{g})$ Detailed view on the post-buckling scenarios depicted in panel (b), showing three post-buckling scenarios A, B, C. (h)-(j) Examples of the post-buckling configurations for end torque of: $20000 \mathrm{Nm}, 45000 \mathrm{Nm}$ and $60000 \mathrm{Nm}$. In each post-buckling scenario there are four shapes marked $A_{i}, B_{i}, C_{i}$, that correspond to branches depicted in panel $(\mathrm{g})$. 

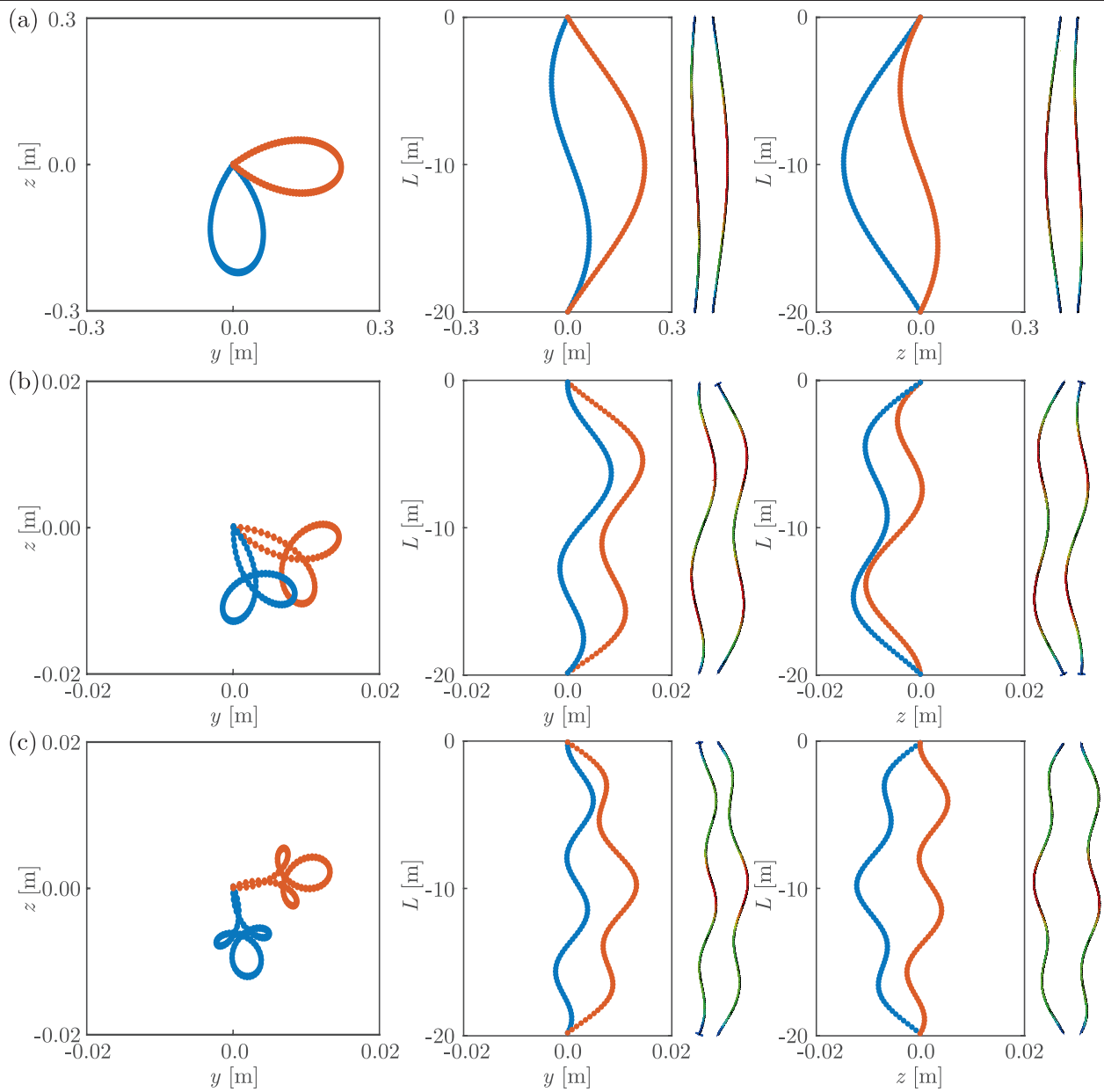

Figure 3. Comparison between coexisting torsional buckling modes (blue and orange curves) from the FE model (90 elements) and the FE modal analysis performed in ABAQUS. (a) $M_{\text {end }}=15814.28 \mathrm{Nm}$ vs $M_{\text {endABQ }}=15822 \mathrm{Nm}$, (b) $M_{\text {end }}=33712.5 \mathrm{Nm}$ vs $M_{\text {endABQ }}=33716 \mathrm{Nm}$, (c) $M_{\text {end }}=52858.2 \mathrm{Nm}$ vs $M_{\text {endABQ }}=52860 \mathrm{Nm}$.

coexistence region is much smaller. In general, this means that in these regions depending on the initial conditions, one can observe two qualitatively different torsional buckling states. In Figure $2(\mathrm{~h})-(\mathrm{j})$, we depict examples of 3D configurations from all considered post buckling scenarios (1-3) for end torque of $20000 \mathrm{Nm}, 45000 \mathrm{Nm}$ and $60000 \mathrm{Nm}$ respectively, as well as corresponding projections on $(y, z)$ plane. As described above, in each scenario, the four branches are symmetric to each other with respect to the origin.

\section{Conclusions}

In this paper we focus on FE modelling of helical buckling of long thin rods, subjected to end torque. In order to achieve a robust method for predicting the critical buckling loads, as well as to follow the post buckling configurations, we modify a standard nonlinear beam element [2] to include the coupling between torsional, axial and lateral DOF. The results 
from the proposed model are verified using static analysis with load stepping, that allows us to obtain an excellent agreement with results from modal buckling analysis in ABAQUS (using higher dimension FE elements). Additionally, the evolution of the post buckling states is investigated, revealing the regions with the co-existing torsional buckling states.

\section{References}

[1] A.R. Champneys and J.M.T. Thompson, Proceedings of the Royal Society of London. Series A: Mathematical, Physical and Engineering Sciences, 452, 2467-2491 (1996)

[2] J.S Przemieniecki, Theory of Matrix Structural Analysis, New York, McGrawHill,(1968)

[3] O.C Zienkiewicz, The Finite Element Method, New York, McGraw-Hill, (1977)

[4] J.F Doyle, Nonlinear Structural Dynamics Using FE Methods, New York, Cambridge University Press, (2015)

[5] X.C. Tan and P.J. Digby, International Journal of Solids and Structures, 30(19), 2675$2691(1993)$

[6] C. Sun and S. Lukasiewicz, Journal of Petroleum Science and Engineering, 50(1), 78-82 (2006) 